\title{
In situ magnetoresistance measurements of ferromagnetic nanocontacts in the Lorentz transmission electron microscope
}

\author{
T. Haug, * K. Perzlmaier, and C. H. Back \\ Institut für Experimentelle und Angewandte Physik, Universität Regensburg, D-93040 Regensburg, Germany
}

(Received 24 October 2008; published 13 January 2009)

\begin{abstract}
We report on in situ magnetoresistance measurements of a 30-nm-wide ferromagnetic nanocontact with simultaneous magnetic imaging in the Lorentz transmission electron microscope. The magnetoresistive measurements are correlated with the micromagnetic configuration of the sample. This allows us to assign characteristic features in the magnetoresistance curves to different magnetic configurations of the sample. From our experiments we can conclude that the micromagnetic configuration of the whole sample-not only the nanocontact region-has to be taken into account for the interpretation of magnetoresistive effects or hysteresis loops. Micromagnetic simulations were performed which confirm the experimental results.
\end{abstract}

DOI: 10.1103/PhysRevB.79.024414

PACS number(s): 68.37.Lp, 75.47.-m, 75.60.Ch, 75.70.Kw

Magnetoresistive (MR) effects in ferromagnetic materials can have different physical reasons. The magnetoresistive behavior of ferromagnetic films or laterally extended structures can be attributed to different MR effects such as anisotropic magnetoresistance (AMR) (Ref. 1) or domain-wall magnetoresistance (DWMR). ${ }^{2,3}$ In nanostructured magnetic systems the situation is often complicated by dipolar effects, giving rise to shape anisotropy. Multidomain configurations may occur which make the analysis of magnetoresistive effects in these samples more difficult. Hence, measurements on ferromagnetic nanocontacts are sometimes discussed controversially, in particular, in nanosized magnetic contacts. ${ }^{4-8}$ For this reason it is highly desirable to link magnetoresistive measurements with high-resolution magnetic imaging.

In order to shed light on the magnetoresistive processes in a ferromagnetic nanocontact and in particular on the contribution of a magnetic domain wall to the resistivity, this paper presents in situ MR measurements of Permalloy (Py, $\mathrm{Ni}_{80} \mathrm{Fe}_{20}$ ) nanocontacts in the Lorentz transmission electron microscope (TEM) (Ref. 9) with simultaneous magnetic imaging. Two different imaging techniques were used, the Fresnel technique ${ }^{10}$ which allows the imaging of magnetic domain walls and the differential phase contrast (DPC) method $^{11}$ which achieves a lateral resolution of about $10 \mathrm{~nm}$ or below ${ }^{12,13}$ and is sensitive to the magnetization within the domains.

Py structures of $20 \mathrm{~nm}$ thickness and about $14 \mu \mathrm{m}$ length were fabricated as shown in Fig. 1(a). All samples were prepared on electron transparent membranes with electron-beam lithography and thermal evaporation. The nanocontact is located in the middle of the structure. On its right side it is connected to a wider stripe (WS) of $1.2 \mu \mathrm{m}$ width, whereas the stripe on the left side has a width of only $500 \mathrm{~nm}$. An external magnetic field is applied parallel to the structure axis ( $y$ direction), and the MR measurement is carried out parallel to the field direction along the $y$ axis.

Due to shape anisotropy, the sample will always tend to align its magnetization parallel to the structure axis. Thus, the magnetization is expected to be parallel or antiparallel to the $y$ direction and therefore to the current path. For this reason, this sample geometry may be suitable to avoid sizable AMR effects that could superimpose the measurements.
Consider the structure saturated along the $y$ direction by a strong external field. When the field is reduced to zero, the magnetization of the sample should more or less stay unchanged. Increasing the field in the opposite direction will result in a switching process until the structure is saturated along the negative $y$ direction. Due to the lower shape anisotropy, the WS is expected to switch its magnetization direction at a lower opposite field than the narrower stripe (NS). This implies that in a certain field range, the WS and the NS are magnetized along opposite directions, and a two-domain state with a magnetic domain wall in the constriction area may be formed. Thus, one should be able to investigate the contribution of a domain wall which is located in a nanoconstriction to the resistivity of the structure.

In the following, we want to discuss the in situ MR measurements of the 20-nm-thick Py structure with 30-nm-wide constriction shown in Fig. 1(b). Figure 2 shows the magnetoresistance measurements of the sample normalized to the

(a)

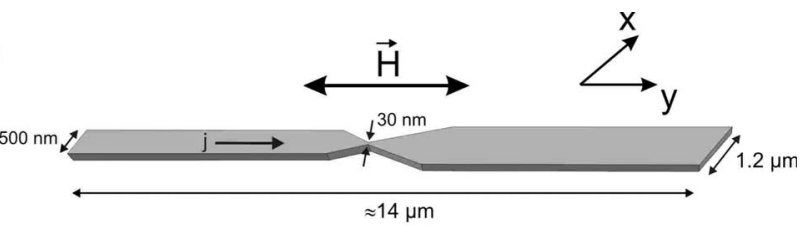

(b)

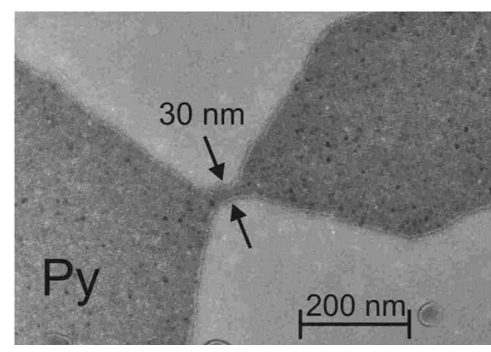

FIG. 1. (a) Sketch of the measured 20-nm-thick Py structure. A 30 -nm-wide constriction in the middle of the sample separates a wider stripe $(1.2 \mu \mathrm{m})$ from a narrower one $(500 \mathrm{~nm})$. An external field $\vec{H}$ is applied parallel to the long axis of the structure; the resistance measurement is done along the field direction at the ends of the sample. (b) TEM image of the contact region of a sample with $30 \mathrm{~nm}$ constriction. 


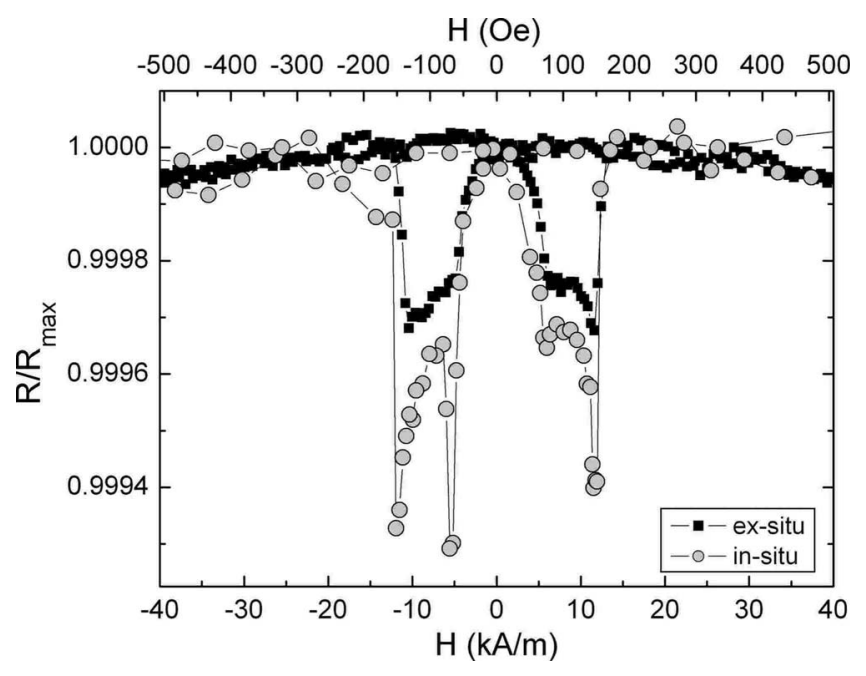

FIG. 2. Ex situ (black squares) and in situ (gray circles) measurements of a 20-nm-thick Py structure with 30-nm-wide constriction.

maximum resistance value of each measurement. The black squares show the data of the ex situ measurement in an external measuring setup; the gray circles represent the in situ measurement in the Lorentz TEM.

We would like to point out that the difference in magnitude of the resistance drop at low fields for the two measurements is due to the normalization procedure. The measurements are done at slightly different temperatures and with a different alignment of the measurement setup with respect to the external magnetic field, and thus the total resistance values can differ from each other. The focus of this paper is on the particular features in the MR curves. At first sight the curves show similar features. Let us first focus on the field sweep from -40 to $+40 \mathrm{kA} / \mathrm{m}$. The resistance stays nearly unchanged when the magnetic field is reduced from saturation to remanence. At a field value of about $4 \mathrm{kA} / \mathrm{m}$, the resistance drops and is reduced further up to a field of about $12 \mathrm{kA} / \mathrm{m}$. Then the resistance jumps back to approximately the saturation value and remains at this value until the field reaches its maximum value of $40 \mathrm{kA} / \mathrm{m}$.

The only characteristic deviation from this behavior in the in situ measurement occurs in the field sweep from $+40 \mathrm{kA} / \mathrm{m}$ down to $-40 \mathrm{kA} / \mathrm{m}$. At the resistance drop around $-4 \mathrm{kA} / \mathrm{m}$, the in situ curve shows a strong negative peak which is visible neither in the ex situ data nor in the opposite direction of the in situ measurement. The reason for this unexpected peak may be due to magnetization processes in the nanoconstriction. A closer investigation of the Lorentz images which was done during the in situ measurement will give more details about the micromagnetic behavior of the sample which is responsible for this effect.

In these measurements, only negative resistance changes can be observed. This already excludes the possibility of a sizable domain-wall magnetoresistance effect which is expected to give a positive resistance contribution. ${ }^{14}$ In addition, the magnetoresistive effect is in the range of only $0.06 \%$ and thus very small. Magnitude and sign suggest that the observed resistance changes are solely due to AMR. Furthermore, another conspicuous fact in Fig. 2 is that the resis-

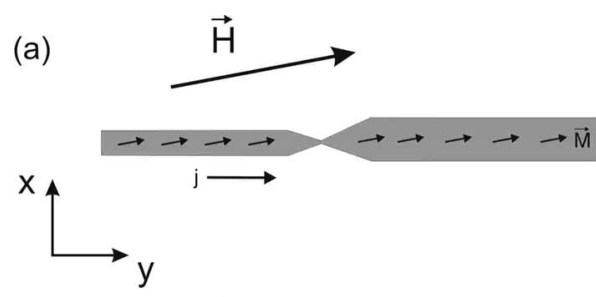

(b)

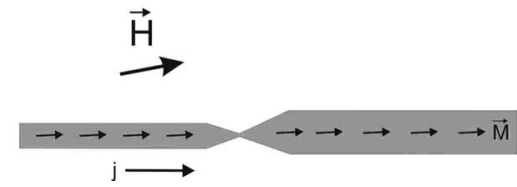

FIG. 3. Sample magnetization $\vec{M}$ for an applied external field $\vec{H}$ that is not aligned exactly parallel to the structure axis ( $y$ direction). (a) The magnetization follows the field direction for strong fields. (b) For weaker fields, $\vec{M}$ can turn along the structure axis due to shape anisotropy.

tance first increases when the external field is reduced from saturation. When only AMR is operative, the resistance should have a maximum when the sample is saturated due to the fact that the magnetization is then aligned exactly parallel to the current direction. In the measurements shown in Fig. 2 , the field direction is probably not aligned exactly parallel to the structure axis ( $y$ direction), as exaggeratively illustrated in Fig. 3. This can be an artifact of the preparation procedure because the sample can only be aligned with an accuracy of about $3^{\circ}$ with respect to the field direction. As a result, the strong field in saturation forces the magnetization to deviate from the $y$ axis and thus from the current direction [Fig. 3(a)], and as a consequence the resistance value is not at its maximum. ${ }^{1}$ With decreasing field, the magnetization will turn toward the $y$ axis due to the shape anisotropy [Fig. 3(b)], which consequently increases the resistance.

Figure 4(a) again shows the MR measurement of the 30nm-wide Py nanocontact shown in Fig. 2. It focuses on the field sweep from -40 to $+40 \mathrm{kA} / \mathrm{m}$, which shall be called the upsweep to simplify matters. The corresponding in situ measuring data are displayed as light gray circles. The darkgray filled circles of the in situ data represent the points where Lorentz images were taken. The corresponding Fresnel images are displayed in Fig. 4(b), whereas the DPC images are shown in Fig. 4(c). The gray arrow indicates the magnetization direction displayed in the DPC images. Dark parts of the sample represent areas where the magnetization points along the negative $y$ direction; bright areas indicate the magnetization pointing along the positive $y$ direction. From $-40 \mathrm{kA} / \mathrm{m}$ up to the remanent state, the resistance stays unchanged. In the Fresnel images [(i)-(iii)], it can be seen that the structure is saturated and the edge fringes indicate that the magnetization points along the negative $y$ direction. At a field of $4 \mathrm{kA} / \mathrm{m}$ [image (iv)], the first signs of a magnetic domain on the right-hand side of the constriction can be seen. The Fresnel image shows the appearance of a bright and a dark wall in the WS, and the corresponding DPC image exhibits a light bright shadow in this region. This domain enlarges with increasing field and can be observed very well both in the Fresnel and in the DPC image corresponding to data point $(\mathrm{v})$ at $6 \mathrm{kA} / \mathrm{m}$. As the magnetization in 

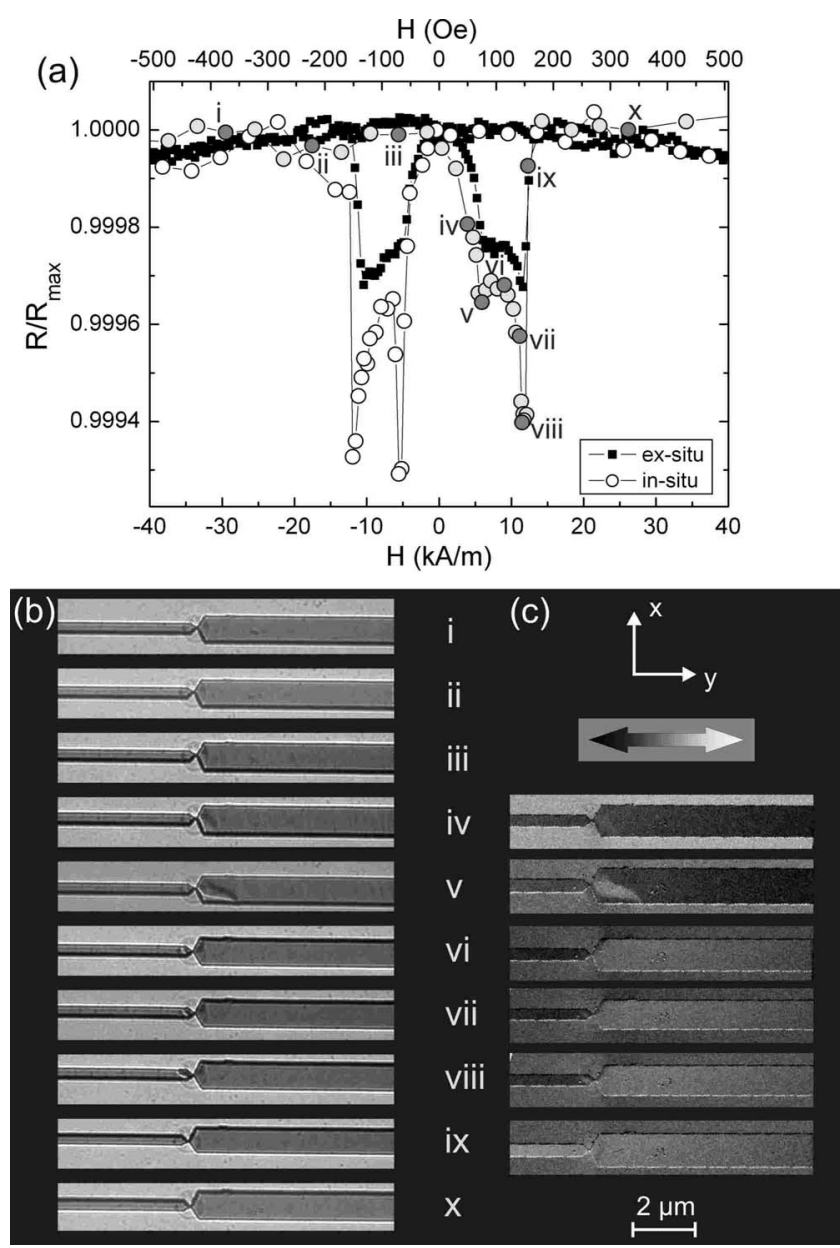

FIG. 4. (a) Ex situ (black squares) and in situ (white/gray circles) MR measurements of Fig. 2. The dark-gray filled circles represent measuring points where Lorentz images were taken during a field sweep from $-40 \mathrm{kA} / \mathrm{m}$ down to $+40 \mathrm{kA} / \mathrm{m}$ (from -500 to $+500 \mathrm{Oe}$ ). (b) Fresnel and (c) DPC images corresponding to the dark-gray measuring points. The gray scale arrow indicates the magnetization direction displayed in the DPC images.

Py never changes abruptly but varies steadily, the appearance of a magnetic domain always involves the appearance of a magnetization component perpendicular to the saturation direction. In this case, the magnetic domain causes an increase in the magnetization component in the $x$ direction and consequently a respective decrease in the $y$ component. Hence, the AMR theory explains the reduction in the resistance with the appearance of the magnetic domain.

When the external field is increased further, the magnetization of the WS switches to the opposite direction whereas the NS stays magnetized along the negative $y$ direction, as can be seen in image (vi) at $8.6 \mathrm{kA} / \mathrm{m}$. Thus, the magnetic domain in the WS disappears, and the resistance is slightly increased. However, it does not reach its starting value which is due to a magnetic domain that appears in the NS on the left-hand side of the constriction. It can be seen in the DPC image as a slight bright shadow at the left of the constriction; the corresponding domain walls are visible in the Fresnel image. In addition, a small domain is pinned at the left upper edge of the WS. This domain disappears when the applied

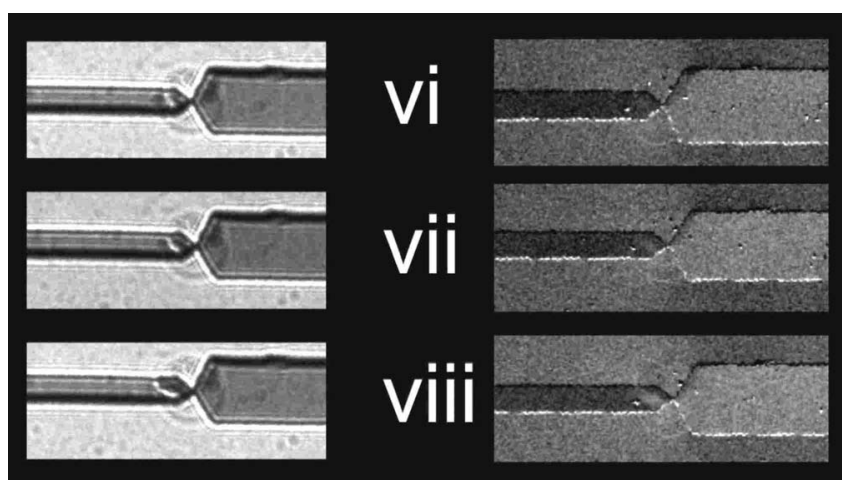

FIG. 5. Enlarged view of the contact region of the Fresnel (lefthand side) and DPC (right-hand side) images (vi)-(viii) from Fig. 4.

field is increased to $12 \mathrm{kA} / \mathrm{m}$ [images (vii) and (viii)], whereas the magnetic domain in the NS is enlarged. Although this domain is very small, it is located directly in the current path, and so it decreases the resistance to a minimum value. Figure 5 shows the magnetic domain in the NS in an enlarged view of images (vi)-(viii) from Fig. 4. With the switching of the magnetization in the NS at $12.4 \mathrm{kA} / \mathrm{m}(155$ Oe) [image (ix) in Fig. 4], all magnetic domains disappear from the structure which is then saturated along the positive $y$ direction. Hence, the resistance jumps back to approximately the starting value and is not changed by the application of higher fields [image $(\mathrm{x})$ ].

The magnetoresistance measurement of the field sweep in the opposite direction from +40 down to $-40 \mathrm{kA} / \mathrm{m}$, which is displayed as light gray circles in Fig. 6(a), shows similar behavior. The Fresnel images taken during the MR measurement are shown in Fig. 6(b); the DPC images are displayed in Fig. 6(c). The corresponding data points in the MR curve are marked as dark-gray circles. This field sweep shall now be called the downsweep. Let us first consider only the region around the nanoconstriction. All the processes in the constriction region happen in analogy to the upsweep shown in Fig. 4, and the resistance behavior of the downsweep can be explained in the same way. However, as the downsweep shows an extraordinary large peak at $-5.5 \mathrm{kA} / \mathrm{m}$, there must be some differences in the remagnetization processes of the two field sweeps. Focusing on images (iv) and (v) of each measurement, we find a magnetic domain appearing in the downsweep in Fig. 6 at the right end of the structure. This domain does not appear in the upsweep in Fig. 4. Consequently a magnetic domain at the end of the sample-and not in the constriction region as first assumed-is responsible for the large resistance decrease in the downsweep at $-5.5 \mathrm{kA} / \mathrm{m}$. The magnetoresistive behavior of both the upsweep and the downsweep can be explained by AMR theory.

In addition to the measurements, micromagnetic simulations were performed using the software package LLG. ${ }^{15}$ It permits the calculation of the magnetoresistive behavior of the sample. The simulations were done using the standard parameters for Py such as the exchange constant $A_{\text {exch }}=13$ $\times 10^{-6} \mathrm{~J} / \mathrm{m}$ and the saturation magnetization $M_{s}=860$ $\times 10^{3} \mathrm{~A} / \mathrm{m}$ with a cell size of $5 \mathrm{~nm}$; the damping constant $\alpha$ was scaled up to shorten computing time. As the sample observed in the experiment was rather large, simulations 

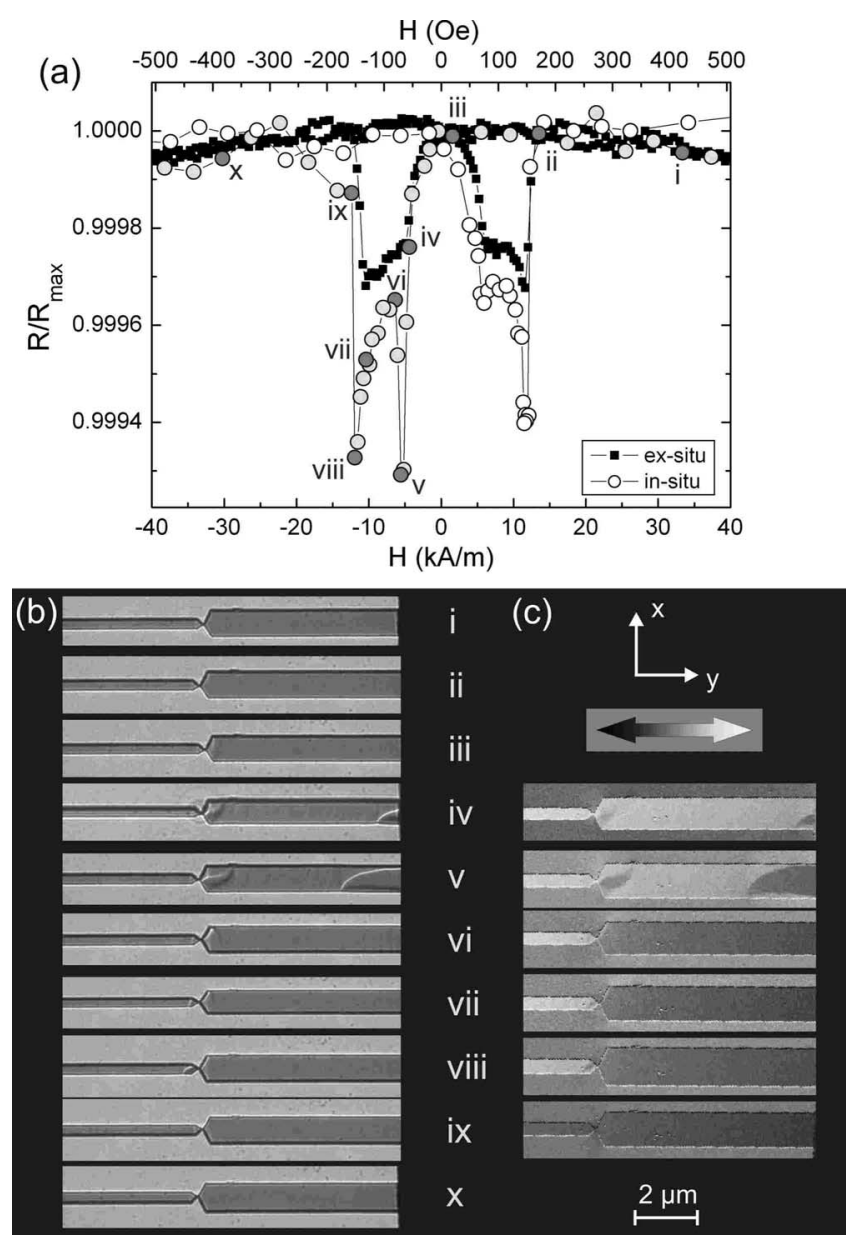

FIG. 6. (a) Ex situ (black squares) and in situ (white/gray circles) MR measurements of Fig. 2. The dark-gray filled circles represent measuring points where Lorentz images were taken during a field sweep from +40 to $-40 \mathrm{kA} / \mathrm{m}$ (from +500 to -500 Oe). (b) Fresnel and (c) DPC images corresponding to the dark-gray measuring points. The grayscale arrow indicates the magnetization direction displayed in the DPC images.

with equal sample dimensions are not possible due to the limited computing power. Thus the WS as well as the NS is shortened, and the simulation only focused on the constriction area. However, the geometrical shape in the contact region is equal to the structure measured in the experiments. Figure 7(a) compares the simulated MR curve (black line) with the previously discussed experimental data. The images of the simulated magnetization distribution are shown in Fig. 7(b). The corresponding resistance values are marked with black arrows in the MR curve in Fig. 7(a). The double arrow at the bottom of the figure indicates the displayed magnetization direction. Bright areas are magnetized along the positive dark areas along the negative $y$ direction. Generally, the remagnetizing process of the simulated structure is comparable to the one measured in the experiment. The sample is saturated along the positive $y$ direction at $40 \mathrm{kA} / \mathrm{m}$. At the sample edges, the first signs of magnetic domains are visible. These domains enlarge with decreasing field down to remanence (images 1-4). The domain in the WS on the right-hand side of the constriction shows similarities to the experimen-

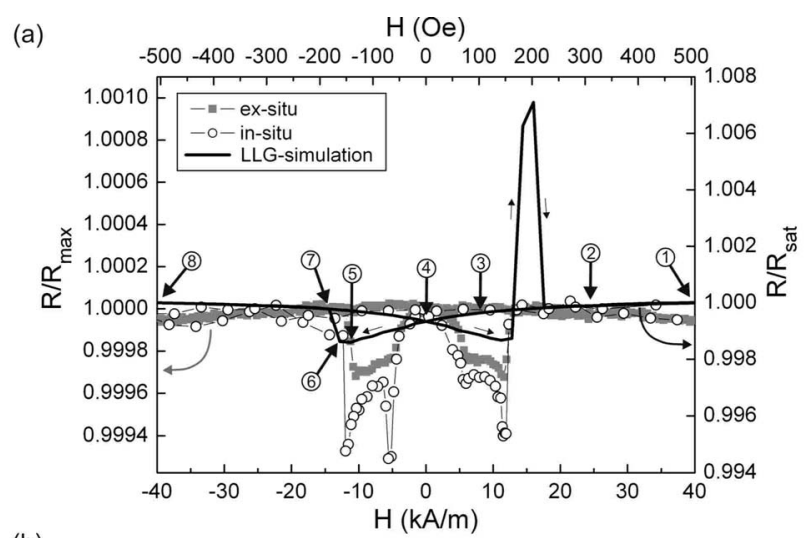

(b)

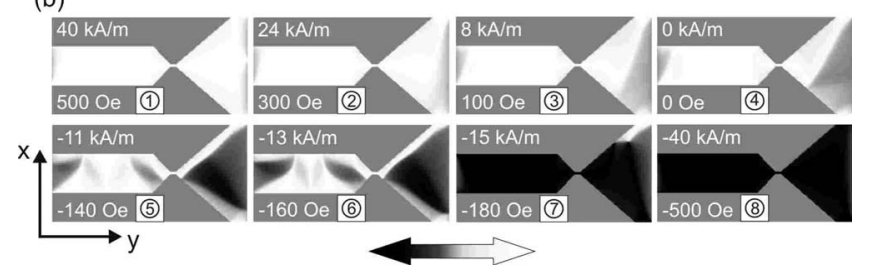

FIG. 7. (a) Comparison of the experimental data (gray squares and white circles) with the MR curve obtained from the LLG simulation (black line). (b) Simulated images of the magnetization distribution within the field sweep from +40 to $-40 \mathrm{kA} / \mathrm{m}$ (from +500 to $-500 \mathrm{Oe}$ ). The corresponding points in the MR curve are marked with black arrows in (a). The double arrow at the figure bottom indicates the displayed magnetization direction.

tally observed domains in images (iv) and (v). However, the structure observed in the experiments has a large shape anisotropy, which suppresses the switching of the WS and thus the appearance of this domain to a small field range of about $3 \mathrm{kA} / \mathrm{m}$. In contrast to that, the reduced length of the WS in the simulations results in the fact that the switching process happens in a broader field range from $40 \mathrm{kA} / \mathrm{m}$ to about $-11 \mathrm{kA} / \mathrm{m}$. This explains the slow but continuous reduction in the resistance value and the differences between the experimental and the simulated MR curves.

At $-11 \mathrm{kA} / \mathrm{m}$ (image 5) and $-13 \mathrm{kA} / \mathrm{m}$ (image 6), the WS has switched its magnetization direction mainly along the negative $y$ direction and the NS on the left-hand side stays more or less along the positive $y$ direction. On the left-hand side of the constriction, a magnetic domain appears as was also observed experimentally in images (vi)-(viii) in Figs. 4 and 6 . The resistance value is now at its minimum. It jumps back to a larger value at about $-15 \mathrm{kA} / \mathrm{m}$, where the magnetization of the NS turns to the negative $y$ direction and the sample is nearly saturated (image 7). This effect of $\approx 0.09 \%$ has the same order of magnitude as the experimentally measured MR. With the displacement of the last domain remaining in the structure, the resistance value increases until it reaches the starting value at $-40 \mathrm{kA} / \mathrm{m}$ (image 8 ).

The remagnetization loop from -40 to $+40 \mathrm{kA} / \mathrm{m}$ shows a similar behavior as described above for the opposite field sweep. This can be seen in the images of the simulated magnetization distribution in Fig. 8(b). However, there is one significant exception when both the WS and the NS have switched their magnetization direction from the negative (dark areas) to the positive (bright areas) $y$ direction. Image 6 
(a)

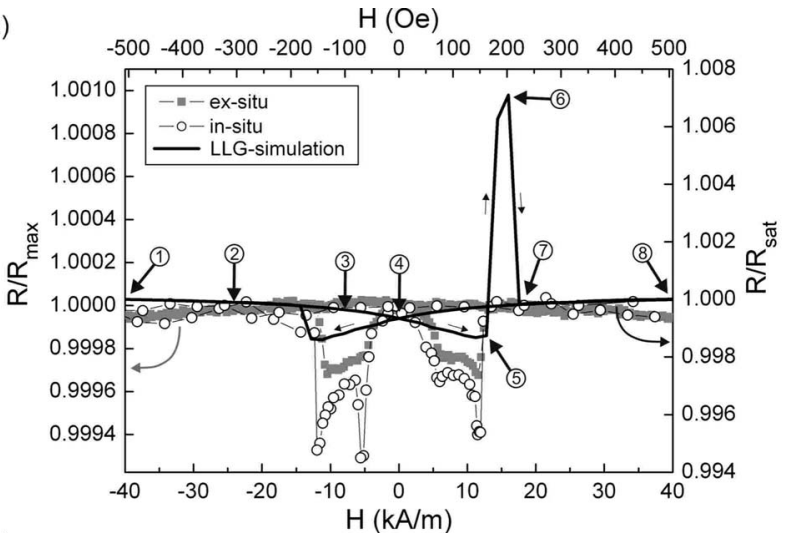

(b)

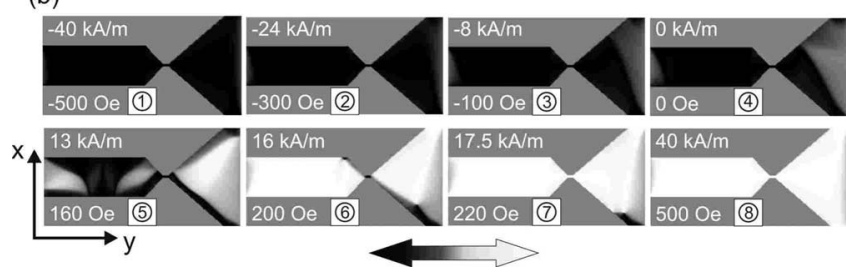

FIG. 8. (a) Comparison of the experimental data (gray squares and white circles) with the MR curve obtained from the LLG simulation (black line). (b) Simulated images of the magnetization distribution within the field sweep from -40 to $+40 \mathrm{kA} / \mathrm{m}$ (from -500 to $+500 \mathrm{Oe})$. The corresponding points in the MR curve are marked with black arrows in (a). The double arrow at the bottom of the figure indicates the displayed magnetization direction.

shows the magnetization distribution at $16 \mathrm{kA} / \mathrm{m}$. Both sides of the nanoconstriction are displayed as bright areas, which is due to the fact that they already switched their magnetization along the positive $y$ direction. The constriction itself, however, is still black, and so it is still magnetized along the negative $y$ direction. Thus, two very narrow domain walls are generated at the ends of the constriction. As the magnetization obviously changes rapidly from the positive to the negative $y$ direction or backward, these two walls lead only to a negligible AMR contribution. Instead, the resistance value is abruptly increased, which can only be explained by the positive resistance contribution due to the DWMR of the two walls. ${ }^{2,3,14}$ With a field of $17.5 \mathrm{kA} / \mathrm{m}$ (image 7 ), also the magnetization in the nanoconstriction has changed its direction into the positive $y$ direction, and with the disappearance of the domain walls the resistance jumps back to the "normal" AMR curve. However, the resistance change is only about $0.7 \%$ and thus very small compared to the large magnetoresistive effects measured in references. ${ }^{4-7}$

The LLG simulations that are shown in this paper were calculated at a temperature of $T=0 \mathrm{~K}$. This may explain why the experimental measurements do not show a positive DWMR, as they were all done at room temperature. In the TEM images no indication of a pinned magnetic domain in the constriction could be found. It seems that the magnetization displayed in image 6 of Fig. 8(b) represents a stable state only at low temperatures which can be depinned by thermal excitations and is therefore only stable in a small field range at room temperature. The experimental MR curves were carried out with a field increment of $400 \mathrm{~A} / \mathrm{m}$. For this reason, the positive peak cannot be reproduced with measurements at room temperature. Apart from this, it is possible that the pinning potential of this metastable state is smaller than the thermal energy at room temperature, and in this case this magnetization distribution cannot exist at room temperature.

In conclusion, we discussed ex situ and in situ MR measurements on a 20-nm-thick Py structure with a 30-nm-wide constriction. The ex situ and in situ measurements show good agreement; quantitative differences can be attributed to artifacts due to normalization. The DPC and Fresnel images that were taken during the in situ MR measurement give a detailed insight into the magnetization distribution of the sample during remagnetization. The magnetoresistive behavior was correlated with the observed micromagnetic configuration. All magnetoresistive effects can be attributed to AMR, and neither a ballistic magnetoresistance effect nor a positive domain-wall contribution to the MR could be measured.

The simulated MR curve reproduces the experiments very well; deviations can be explained by the different shape anisotropies of the samples and by the fact that the experiments were done at room temperature, whereas the simulations were calculated at $T=0 \mathrm{~K}$. This may also be the reason why the simulations show a positive DWMR, whereas the experiments could not show any comparable effects. All things considered, the simulated MR curve confirms the statement that all magnetoresistive effects measured in the experiments can be attributed to AMR.

The appearance of a large peak in one of the MR measurements was correlated with a magnetic domain that appeared at the edge of the structure, far away from the constriction. Our experiments demonstrate that it is very important to take also the whole sample into account for the interpretation of hysteresis loops or MR measurements and not only the region of interest.

We would like to thank D. Weiss for the possibility of preparing our samples in his clean room. Furthermore, the authors acknowledge M. R. Scheinfein for his support in all questions concerning LLG.

\footnotetext{
*thomas.haug@physik.uni-regensburg.de

${ }^{1}$ T. R. McGuire and R. I. Potter, IEEE Trans. Magn. 11, 1018 (1975).

${ }^{2}$ P. M. Levy and S. Zhang, Phys. Rev. Lett. 79, 5110 (1997).

${ }^{3}$ F. S. Bergeret, A. F. Volkov, and K. B. Efetov, Phys. Rev. B 66,
}

184403 (2002).

${ }^{4}$ N. Garcia, M. Munoz, and Y.-W. Zhao, Phys. Rev. Lett. 82, 2923 (1999).

${ }^{5}$ N. Garcia, M. Munoz, G. G. Qian, H. Rohrer, I. G. Saveliev, and Y.-W. Zhao, Appl. Phys. Lett. 79, 4550 (2001). 
${ }^{6}$ H. D. Chopra and S. Z. Hua, Phys. Rev. B 66, 020403(R) (2002).

${ }^{7}$ S. Z. Hua and H. D. Chopra, Phys. Rev. B 67, 060401(R) (2003).

${ }^{8}$ R. A. Lukaszew, D. Pearson, and Z. Zhang, J. Vac. Sci. Technol. A 22, 1371 (2004).

${ }^{9}$ J. N. Chapman and M. R. Scheinfein, J. Magn. Magn. Mater. 200, 729 (1999).

${ }^{10}$ J. N. Chapman, J. Phys. D 17, 623 (1984).
${ }^{11}$ J. N. Chapman, I. R. McFadyen, and S. McVitie, IEEE Trans. Magn. 26, 1506 (1990).

${ }^{12}$ T. Uhlig and J. Zweck, Ultramicroscopy 99, 137 (2004).

${ }^{13}$ T. Haug, A. Vogl, J. Zweck, and C. H. Back, Appl. Phys. Lett. 88, 082506 (2006).

${ }^{14}$ B. Leven, U. Nowak, and G. Dumpich, Europhys. Lett. 70, 803 (2005).

${ }^{15}$ M. R. Scheinfein, http://llgmicro.home.mindspring.com (2003). 